\title{
The Parents' Perception of Nursing Support in their Neonatal Intensive Care Unit (NICU) Experience
}

\author{
Amani F. Magliyah \\ College of Public Health and Health Informatics, \\ King Saud bin Abdulaziz University for Health Sciences, \\ Saudi Arabia
}

\begin{abstract}
NICU is an environment that has many challenges in information receiving and understanding. The infants that are cared for might have serious and complex medical problems. For Parents the NICU experience is filled with stress, fear, sadness, guilt and shock of having a sick baby in NICU. The aim of this research was to explore and describe parents' experience when their infant is admitted to the NICU. And assess their perception of nursing support of information provision and according to their emotional feelings. This study was undertaken at Neonatal Intensive Care Unit in King Abdulaziz Medical City (KAMC), Jeddah, Saudi Arabia which is part of National Guard Health Affairs (NGHA) organization in the kingdom. The study utilized a self-report questionnaire with likert scale measurement and telephone interview with closed questions. One hundred and four parents agree to be the part of study and provided their consent to include their children in the study. The majority of respondents were mothers $(76 \%)$, the remaining $(24 \%)$ from the total sample were Fathers. All their infants have been admitted to the NICU at 2014. Many parents did not able to receive enough information easily from the unit; most of them found the information by nurses was difficult to understand. The majority of parent's perceived high stress and anxiety level according to this information. Also, Most Parents was not agreed about the nurses' support towards their emotional feeling and care. Additional finding indicate that a decrease in support level being associated with an increase in stress and anxiety level. In order to provide a high level of support and decrease the level of stress, there is a need for developing support strategies. One strategy is through a technology to develop an automatic daily summary for parent.
\end{abstract}

Keywords-parents; stress; anxiety; NICU; nurse support; neonate; infant

\section{INTRODUCTION}

A neonatal intensive care unit (NICU) is specializing in the care of sick or early newborn infants [2]. Inside this unit, all of the critical life support, physiological monitoring and medical attention are provided twenty-four hours a day. The infants that are cared for might have serious and complex medical problems [6]. For parents, the NICU experience is an unanticipated journey filled with stress, emotional turmoil, strains on relationships and sometimes depression [2]. They rarely feel safe from the fear and uncertainty of the problems that can occur while the child is in care; the sequence of events in the NICU journey has many unexpected ups, downs, and turns of event [6]. Usually medical staff provides a large amount of information to the parents. Parents have to come to

\author{
Muhamamd I. Razzak \\ College of Public Health and Health Informatics, \\ King Saud bin Abdulaziz University for Health Sciences, \\ Saudi Arabia
}

terms with information that they are not familiar with and have to deal with the emotional impact of the information presented to them [2]. However, the provision of clear and summarized information is important in giving parents a sense of hope and a feeling of involvement in their child's care [6]. Therefore, the research question for this study is: Are parents able to get information about their infant and received nursing support according to their feelings in their NICU experience?

Much of the literature confirms that there is a need for improving interventions, provide consistent information sharing, and high level of support for parents in the environment of NICU; to reduce parental stress, anxiety and negative feelings, improve family-centered care, and to increase parent's satisfaction and involvement in the NICU [1, $4,5,9]$. Parents of infants admitted to the NICU will experience stress, depression, anxiety, and feelings of powerlessness, and hopelessness [9]. They used to cope with this stress via preexisting support systems of family and friends [3]. Lam et al. [4] findings indicate that parents of infants in the NICU had a lower level of stress if they perceived a high level of support, and there is a need for improving the nursing support strategies in providing parents with clear and updated information. According to Grosik et al. [2] once stressors have been identified, then interventions can be developed to improve the family-centered approach to care. It has been shown that there is a need to develop local interventions to decrease stress and enhance parents' abilities and understanding of their infant [5]. In addition to, providing holistic, family-centered, developmentally supportive care and open communication with parents in this stressful experience [9]. A 2011 study [1] found that because of an intervention the parental stress during the NICU stay was not reduced, while satisfaction with information and preference for involvement were both increased.

One means of achieving the intervention and support for parents is through developing software that generates summaries of medical data about babies in NICU using natural language processing (NLP). Research by Mahamood and Reiter [6] showed that all parents preferred texts generated with the effective strategies, and the key finding was that the use of these affective strategies might be appropriate whenever an NLP system is communicating emotional sensitive information to a non-expert recipient. Although this research provided a solution using NLP technology, it does not examine whether this software can support parents in Arab countries 
and gaining their preference through generating daily summary reports. Some researchers [2, 5, 9] focused on exploring parental stressor and experience in NICU without specifying their perception of nursing support and information provision. While Franck et al. [1] measured the effect of an intervention but limited on parents stress, confidence and competence. The research by Lam et al. [4] measured the parents support provided by nurse and correlated with parents stress, it was similar to our research question but without determining the stress levels and correlated with nursing support level. Finally, these studies have not been applied in the Saudi Arabia environment. This research will present information describing the current situation in NICU parental experience, and the level of parent's perception of the information support by nurse; that will help NICU to take appropriate action and to develop an intervention to improve support strategies, promote family centered care and developmentally supportive care.

\section{OBJECTIVES}

NICU is an environment that has many challenges in information receiving and understanding. Parents feeling of stress, fear, sadness, guilt and shock of having a sick baby in NICU might mean that parents will not be able to process large amounts of unfamiliar information. The aim of this research was to explore and describe parents' experience when their infant is admitted to the NICU, in addition to their perception of nursing support of information provision and according to their emotional feelings. This information will help healthcare professionals to understand the parental experience in the NICU, and to develop interventions that improve supporting strategies. The following was the specific objectives; to investigate current parents experience in NICU with information provision by a medical staff; to explore the parent's ability to get needed information in NICU; and to explore parent's perception of nursing support according to their emotional feelings.

\section{METHODLOGY}

The participant (parents) consent were taken along with the survey questioner in order to include in the study.

\section{A. Study Area and Setting}

This study was conducted at National Guard Health Affairs (NGHA), Jeddah. NGHA is one of the largest health organizations in the Kingdom of Saudi Arabia. The Saudi Arabian National Guard Health Affairs aim to provide the highest quality of primary, include all aspects of care and patients treatment. In addition to, the increase of awareness among members secondary and tertiary healthcare services whilst ensuring efficiency and proper utilization of available resource [7].

King Abdulaziz Medical City (KAMC) Jeddah is part of NGHA. It is offers medical care services on advance level for Saudi nationals in the Western Region, and these services of society towards the prevention of diseases. This city makes effort to do medical initiatives and participation at all levels of local ,regional and international. Since the opening of the hospital in 1402 AH (1982 AD), strenuous efforts have been made to maintain the level of quality of care provided to patients and their advancement, until the hospital evolved and became part of the KAMC in Jeddah [3].

If a newborn baby needs intensive medical attention they are often admitted into a special area of the hospital called the Neonatal Intensive Care Unit (NICU). The NICU provides 24 hours service and only accept infants requiring level II and III specialized care. The unit is staffed with nurse to patient ratio of 1:1-2. It provides a family-centered approach to care; encompassing the parents and sick infant as a single unit. Thus including the family in all decision making and kept well informed and have complete understanding of all patient care activities/procedures. The NICU services provide the best technological evidenced - based treatment and care [8].

\section{B. Study Subjects}

The sample included both male and female parents who have an infant in NICU at KAMC hospital located in Jeddah. In order to observe and get better feeling, the sample was restricted to parents of infants who stayed one full day or more in NICU. The major key part of the sample unit for this study was on Saudi female parents between 18 and 50 years old, and Saudi male parents between 18 and 60 years old. In External validity, we can generate the sample to the target population inside the hospital, because the sample is randomly assigned. It can't be generalized to other population inside the country and to other countries due to the small sample size. Parents provided their consent in order for the children to be included in this study.

\section{Study Design}

The study design was a descriptive design using cross sectional study that has been carried out over a short period (4 month). It was used to estimate the participants perception of nursing support in the NICU experience and their ability to get needed information. No pre-tests and intervention were used.

\section{Sample Size}

The target population was all parents of infant in the NICU located in NGHA hospital at Jeddah. Estimated size of 142 parents included in the medical record at 2014 of NICU in NGHA. Normally, the both parent visit the hospital with infant thus the total population may be increased to include the other partner, but in our case we limited the population size to be as it is in the medical record and included some case where one of parents visit the hospital. The sample size was estimated and it was of 104 parents who agree to be the part of this study and provide their consent to include in the study with $\pm 5 \%$ Precision Levels, where Confidence Level is $95 \%$ and $\mathrm{P}=.05$.

\section{E. Sampling Technique}

Simple random sampling technique was used to select a representative sample of parents of infants admitted in the NICU at KAMC, Jeddah. Each participant was chosen from the medial record of NICU unit during 2014.

Since there are only 59 valid records, the rest participants were chosen when they come to see their infant or for their infant's vaccine. The process continued until sufficient participants have been identified to meet the desired sample size. 


\section{F. Data Collection methods, instruments used, measurements}

The researcher aimed to identify parent's self-reported opinion about their perception of nursing support and to describe their NICU experience with current and traditional information provision. The questionnaire survey contained three parts; the first one was the personal basic information which is name, age, gender and educational level and their consent to include in the study. The second part about the parents experience with information's by nurses in NICU. Last part was about the current nursing support of their negative emotional feelings.

TABLE I. PARENT CHARACTERISTICS $(\mathrm{N}=104)$

\begin{tabular}{|c|c|c|c|c|}
\hline \multicolumn{2}{|r|}{ characteristic } & n $(\%)$ & Mean & $\begin{array}{c}\text { Std. } \\
\text { Deviation }\end{array}$ \\
\hline Gender & $\begin{array}{c}\text { Male } \\
\text { Female }\end{array}$ & $\begin{array}{l}25(24 \%) \\
79(76 \%)\end{array}$ & - & - \\
\hline Age & $\begin{array}{l}<20-30 \\
>30-50\end{array}$ & $\begin{array}{l}48(46 \%) \\
56(54 \%)\end{array}$ & 30.53 & 6.49 \\
\hline $\begin{array}{c}\text { Education } \\
\text { Level }\end{array}$ & $\begin{array}{l}\text { 1. ElementarySchool } \\
\text { 2.HighSchool } \\
\text { 3.UniversityDiploma } \\
\text { 4.UndergraduateUniversity } \\
\text { 5.PostgraduateUniversity }\end{array}$ & $\begin{array}{c}21(20.2 \%) \\
39(37.5 \%) \\
1(1 \%) \\
41(39.4 \%) \\
2(1.9 \%)\end{array}$ & 2.65 & 1.24 \\
\hline
\end{tabular}

The survey was tested, and all questions were revised and it was found that, the personal information part had no modification. The Questions in second part were found to be a little bit confusing, these questions were retyped and rephrased to be clearer. In addition, response categories in questions eight and nine were modified to be in a form of measuring their current feeling in NICU experience when nurses told them information about their preterm infants. Last part was clear to them and no modification was applied. The question number five, ten and twelve in the survey will be used to collect the research variables.

The researcher used a telephone interview that requires the respondent to answer the closed question in the questionnaire. In addition to a self-administered survey technique that requires the respondent to complete the questionnaire by him/herself. These surveys were distributed in-person and responses were collected directly into the questionnaire, which needed to establish a good relationship with participants (parents) and a comfortable flow of questions, in order to ensure that the appropriate data are collected. The researcher wanted to reach a sample size of 104 parents, and assumed that the response rate might be $95 \%$ of the needed sample size. Initial sample size was parents of infants at NICU with lower levels of specialized care at KAMC hospital in Jeddah.

\section{G. Data Management and Analysis}

Data were tabulated on IBM SPSS sheet and have been analyzed using the statistics in the SPSS software for Windows. Discrete data were analyzed using use CI 95\% for the descriptive data to measure the mean and standard deviation of the scores.
A convenience sample of 104 parents agreed to be part of the study and provided their consent in order for the children to be included in the study. Sixteen parents answered to the questionnaire's questions by a phone interviews. And forty four parents answered the questionnaires via self-administered method. The majority of respondents were mothers (76\%), the remaining (24\%) from the total sample were Fathers. All their infants have been admitted to the NICU at 2014.

The characteristic description of the parents is shown in Table 1; the mean age of the participant was about 31 years old; the majority of them were between 30 to 50 years; in the education level most of them had an undergraduate university degree (39\%) from both genders, and a high school level (38\%) where most of them were mothers; $20 \%$ had an elementary school level and all of them were mothers; $2 \%$ of female persons have postgraduate university degree, and only $1 \%$ of males had a university diploma.

The parent's perceived levels of agreement associated with information's provided by Nurse in NICU in addition to the levels of stress are shown in Table 2. The mean score for each perceived rates is shown. The range of scores was from 1-5 (from positive to negative) that indicate Likert scale level of measurement and agreement. The findings present that a total of $51 \%$ of parents disagreed that they can easily get information and help from nurse when they visited or telephoned the unit, while $40 \%$ parents are agreed. $5 \%$ of them rated neutral and $4 \%$ are strongly disagreed. Most of parents $(64 \%)$ are disagreed that they received from nurses enough information daily about their baby progress, however $18 \%$ of parents agreed on that. $9 \%$ of them rated neutral and $9 \%$ are strongly disagree. The percentage of parents who disagreed that they understood what nurses told them about their infants is $59 \%$ giving the most rates, just $23 \%$ are agreed and $1 \%$ was strongly agreed. $10 \%$ of them rated neutral and $7 \%$ are strongly disagreed.

TABLE II. MEAN AND STD. SCORE RELATED TO INFORMATION'S PROVIDED BY NURSE IN NICU

\begin{tabular}{|c|c|c|}
\cline { 2 - 3 } \multicolumn{1}{c|}{} & Mean & $\begin{array}{c}\text { Std. } \\
\text { Deviation }\end{array}$ \\
\hline Easy to get information or help from nurses & 3.18 & 1.02 \\
\hline $\begin{array}{c}\text { Received enough information daily about my baby } \\
\text { progress }\end{array}$ & 3.63 & .88 \\
\hline Understood what nurses told me about my baby & 3.47 & .95 \\
\hline $\begin{array}{c}\text { Stress after nurse's informations about my baby's } \\
\text { condition }\end{array}$ & 1.88 & .37 \\
\hline $\begin{array}{c}\text { Anxiety after nurse's informations about my baby's } \\
\text { condition }\end{array}$ & 1.79 & .49 \\
\hline
\end{tabular}

In the NICU environment the infant's status can be changed frequently, many tests can be performed and may include many treatments. The majority of parents $(85 \%)$ had a high stress level after nurses told them about their infant's condition, while $13 \%$ of them had a very high stress level and only $2 \%$ had a moderate level. Most of parents $(71 \%)$ had a high anxiety level after nurses told them about their infant's condition. While $25 \%$ of them had a very high anxiety level and only $4 \%$ had a moderate level.

The parent's perceived levels of agreement associated with nursing support of parents feelings during the NICU 
experience are shown in Table 3. As previous part the mean score for each perceived rates is shown. Most of parents (68\%) are disagreed that nurses responded to their worries or concerns clearly and $1 \%$ is strongly disagreed. $20 \%$ of them rated neutral and $11 \%$ are agreed. A total of $73 \%$ of parents disagreed that nurses allowed them to be involved in their infant's care, while $21 \%$ are agreed. $4 \%$ of them rated neutral and $2 \%$ are strongly disagreed. The last one was a bout nurse' support in general towards parents their infant's hospitalization. $60 \%$ parents are disagreed, that means the support level was low and $1 \%$ is strongly disagreed that indicate very low support level. $35 \%$ of them rated neutral and only $4 \%$ are agreed and it indicates that they had a high support level.

TABLE III. MEAN AND STD. SCORE RELATED to EMOTIONAL SUPPORT PROVIDED BY NURSE IN NICU

\begin{tabular}{|c|c|c|}
\cline { 2 - 3 } \multicolumn{1}{c|}{} & Mean & Std. Deviation \\
\hline $\begin{array}{c}\text { Nurses responded to my } \\
\text { worries or concerns clearly }\end{array}$ & 3.60 & .69 \\
\hline $\begin{array}{c}\text { Nurses allowed me to be } \\
\text { involved in my baby's care }\end{array}$ & 3.56 & .84 \\
\hline $\begin{array}{c}\text { Nurses' support towards me } \\
\text { during my baby's } \\
\text { hospitalisation. }\end{array}$ & 3.58 & .58 \\
\hline
\end{tabular}

From the results we found that most parents $(n=53,51 \%)$ who disagreed about the given nursing support, received a high stress and it is most percentage in the stress level as shown in Table This means a decrease in support level being associated with an increase in stress level. However, the intersection of the parents who received a high anxiety level and disagreed about the given support was $44 \%$ of them $(n=46)$, and it is lower than the stress. In addition there were some parent's perception of stress and anxiety did not match with their perception of support.

\section{DISCUSSION}

The results of this study have shown that parents of infants admitted to the NICU had a high level of stress, high level of anxiety and a low level of support from nurses according to infant's information. This is an interesting finding that most parents find the experience in the NICU stressful. This study is consistent with earlier research [4]; they found a moderate correlation between stress and support variables, which indicates that "with a high level of nurse support, the stress perceived by the families would be less". Another previous study [2] found that the highest stress scores were in parents observations for their infants in distress or when appearing very ill. Also parents' were most stressful by the noise of alarms and machines located in the unit. Furthermore, they proved that parents were struggle of separation and helplessness from their babies in the NICU [2]. However, Linda \& Trudi [5] have shown that parents were experienced a moderate to high stress levels regarding their infant's stay in NICU. It has been clear that "having an infant in the NICU was an overwhelming experience associated with negative feelings. These included role strain, distress, and emotional pain" [9].
TABLE IV. P.STRESS * NURSESUPPORT CROSSTABULATION, SPSS

Count
\begin{tabular}{|cc|c|c|c|c|c|}
\hline \multirow{4}{*}{} & \multicolumn{3}{|c|}{ NurseSupport } & Total \\
\cline { 2 - 6 } & Agree & Neutral & Disagree & StronglyDisagree & \\
\hline \multirow{3}{*}{ P.Stress } & VeryHigh & 1 & 3 & 9 & 1 & 14 \\
& High & 2 & 33 & 53 & 0 & 88 \\
& Moderate & 1 & 1 & 0 & 0 & 2 \\
Total & & 4 & 37 & 62 & 1 & 104 \\
\hline
\end{tabular}

Many changes could occur frequently in the infant's condition during hospitalization such as surgery and postoperative processes [5]. This study showed that the majority of parents didn't understand the information provided by nurse. In the study done by Linda \& Trudi [5] shown that parent want to be understood by medical staff in the unit, not just by their friends and family. And in their situation they need for empathy to them not a pity from people. In addition, "They wanted clear and accurate information, guidance, understanding and empathy from people around them" [5]. Parents were considered the infant pain as very essential value and they wanted more of information to be involved with their infants, especially during these painful procedures [1].

Lam et al. [4] found that parents who didn't understand their baby's condition were perceived as highly stressful experiences. Also, they found that "some parents did perceive that nursing communication and behavior contributed to their stress, in particular when they felt the nurses did not give them enough information about tests or treatments being undertaken" [4]. This correlate with our result which found some of parents didn't able to get information about their baby's condition and progress easily from NICU. In addition most of them didn't get enough information daily from the nurses. Regarding to these findings lam et al. [4] had mention that in order to help the families cope, the staff in a particular NICU have to develop several strategies to use it in their practice. Linda \& Trudi [5] found that the communication was an essential aspect that parents care about in their experience, in which that the ways of perceiving the communication wither in a good or bad way had impact their stress experience directly. While the communication was recognized as important, parents were needed to understand their infant's status and progress, and the care delivered to them. In addition, "The manner in which staff went about their business was recognized as a potential stressor" [5]. That means when a good communication and education were provided by staff, and parents were involved in the care, this had impact positively on the parents' experience. But when the staff practices or behaviors are inconsistent this was considered as unprofessional and the parental stress level was increased [5].

Our result shown that parents' perception of receiving support from nurses was low, nurses didn't respond to the worries and concerns of most of the parents. Lam et al. [4] said that there is a need for an emotional and psychological support to take in the consideration, and nurses have to provide a level of support to meet with parents need. 
In addition, our study finds that nurses didn't allow parents to be involved in their infant's care." Both mothers and fathers spoke of stress from the separation from their baby. This experience was intensified when the baby was unstable, whilst the mother was in the postnatal ward and for mothers when breastfeeding" [5]. Obeidat et al. [9] found that the mothers was needed to be closed ,near and belong to their babies, and if these needs were achieved, the mothers will have a feelings of responsibility, confidence, and familiarity with their infant . Furthermore, "when parents were involved in infant care, were allowed proximity, communicated clearly and openly, and formed rapport with the nurses, they became more satisfied and confident in their parenting roles"[9]. A previous study by Franck et al. [1] have demonstrated that most of parents were realized that the intensity of their infant's pain was a little high, thus their preference to be involved actively during these procedures was very strong. Also they found that "mothers who participated in a Newborn Individualized Developmental Care and Assessment Program reported feeling closer to their infants but also experienced a higher level of anxiety than mothers who did not participate in the program" [1].

In this study, these findings has leads the researchers to consider a technical way of to develop and provide support for the parents. The reason for thinking about technology rather than other strategies is the natural of NICU's environment." Sometimes the busy workload may contribute to a perceived lack of time available for supportive care, or nurses may even forget that parents are heavily dependent on them for emotional support and parental guidance" [4]. Mahamood and Reiter [6] had presented a system with affective NLG strategies for medical texts generation that developed for parents of pre-term infants. These generated texts are an English summary contains data and information about infants in a NICU, and it serves as stress reduction tool. They found that most of parent preferred using the system, they found it emotionally appropriate, helpful, and the information was understandable [6]. Thus, the generation of such reports for parents was useful and successful. So, further research on technical ways is required in order to increase parental guidance, support and involvement, to achieve parents' satisfaction during the neonatal stay, especially in Arabic countries.

\section{LIMITATION}

In the study where the researcher analyzed and explored the perception of nursing support to parents in NICU experience, the main limitations were the small sample size and the crosssectional study design. Also some of data collection process conducted through surveying the participants in one and busy NICU unit during a short period. In addition, there were a number of threats to internal validity. First, selection bias was likely to be a more significant threat, participant may have been chosen intentionally, or it may not have been possible to randomly assign participants. To avoid this type of a threat, authors used random assignment for Parents' selection so that they were being measured across a range of general and specific criteria such as age, behavior, gender, educational level and morality. Second, the participant reactivity and behavior; most of the parents were tired, depressed, bored, not cooperative and inattentive. Such factors were difficult to control and it could reduce the internal validity. Thus, the authors used mixed method for collecting data and add phone interview method; because the parents feel more comfortable than when they are in the hospital. Also mixed methods used to reach the desired sample size in the small period.

Third, statistical regression was a threat; the scores of individuals on the dependent variable were not only being due to the natural performance of those individuals, but also measurement errors or chance. In addition, the researcher effect that is typically unintentional, but arises because of the personal characteristics of the researcher that influences the choices made during a study, and non-verbal cues that the researcher gives out that may influence the behaviour and responses of participants. Finally, the testing threat was controlled because this was not an experimental study and there was no pretest. Also changes related to time (maturation, history etc.) were not measured because there was no pretest.

\section{RECOMMENDATIONS}

The finding from this research is evidence that we need a solution to improve parents' perceptions about neonatal experience. The authors recommend to develop Automatic Summary Generator System in order to Supporting Parent's at NICU, reduce the stress and anxiety level to an acceptable levels, and provide a support to parent to increase their satisfaction about the support delivered in this environment. The system used natural language processing technique (NLP) and it has used a lot in the creation of e-Health systems especially in generating information [6]. It assists healthcare practitioners in providing information support to doctors and nurses [6]. Beside this, NLP technique is "playing a greater role in providing patients with access to information in a personal form"[6]. In order to implement this system, the authors suggest following the study conducted by Mahamood and Reiter [6], begin with the management plan, then the design, implementation, and evaluation of this system. It have to be adapted it to be suitable to Arabic parents in the Kingdom.

\section{CONCLUSIONS}

One hundred and four parents agree to be the part of study and provided their consent to include their children in the study. The majority of respondents were mothers $(76 \%)$, the remaining $(24 \%)$ from the total sample were fathers. This study showed a high level of stress and anxiety and a low level of support have been perceived by parents in NICU experience. It helps to recognize the importance of establishing support with parents during their infant's hospitalization in the NICU where most of negative feelings occur. The study has also shown that nurses did not provide emotional support to parents. A recommendation from the findings of this study presents that there is a need for an Automatic summary generator for parents to alleviate their stress, anxiety, increase support, and care involvement, as a support strategy that can improve parent's emotional support with care and information.

\section{ACKNOWLEDGEMENT}

The author thanks Ms. Intisar Abdullah, MS.RHIA, and Director Health Information Management Department at NGHA Hospital for her support in data collection through 
providing parent's phone numbers in medical records. This work has been financially supported by King Abdullah International Medical Research Center (KAIMRC) National Guard Health Affair, Saudi Arabia through grant SP14/059.

\section{REFERENCES}

[1] Franck, L. S., Oulton, K., Nderitu, S., Lim, M., Fang, S., \& Kaiser, A (2011). Parent Involvement in Pain Management for NICU Infants: A Randomized Controlled Trial. Pediatrics , 128 (3).

[2] Grosik, C., Snyder, D., Cleary, G. M., \& Breckenridge, D. M. (2013). Identification of Internal and External Stressors in Parents of Newborns in Intensive Care. The Permanente Journal. , 17 (3), 36-41.

[3] King Abdul Aziz Medical City - Jeddah. (n.d.). Retrieved from NGHA http://www.ngha.med.sa/Arabic/MEDICALCITIES/Jeddah/Pages/defaul t.aspx

[4] Lam, J., Spence, K., \& Halliday, R. (2007). Parents' perception of nursing support in the neonatal intensive care unit (NICU). Neonatal, Paediatric and Child Health Nursing , 10 (3).
[5] Linda, S., \& Trudi, M. (2012). Identification of parental stressors in an Australian neonatal intensive care unit. Neonatal, Paediatric and Child Health Nursing, 15 (2).

[6] Mahamood, S., \& Reiter, E. (2011, September). Generating Affective Natural Language for Parents of Neonatal Infants. Association for Computational Linguistics , 12-21.

[7] National Guard Health Affairs Hospitals. (n.d.). Retrieved from Geneva Health: http://www.genevahealth.co.uk/internationalopportunities/middle-east/working-in-saudi-arabia/national-guardhealth-affairs-hospitals.aspx

[8] Neonatal Intensive Care Unit . (n.d.). Retrieved from NGHA: http://www.ngha.med.sa/English/MedicalCities/AlMadinah/NursingSvc/ UnitsServices/Pages/NeonatalIntensiveCareUnit.aspx

[9] Obeidat, H. M., Bond, E. A., \& Callister, L. C. (2009). The Parental Experience of Having an Infant in the Newborn Intensive Care Unit. The Journal of Parental Education , 18 (3), 23-29. 